livraisons

d'Histoire

de l'Architecture

\section{Livraisons de l'histoire de l'architecture}

41 | 2021

L'architecte en son agence

\title{
Les grandes fortunes du cabinet Dauvergne (1850-1919), des conditions du monopole aux limites d'un modèle économique
}

The great fortunes of cabinet Dauvergne (1850-1919), from monopoly conditions to the limits of an economic model

Das Vermögen des Architekturbüros Dauvergne (1850-1919). Von den

Grundlagen eines Monopols zu den Grenzen einer Geschäftsmethode

\section{Olivier Prisset}

\section{OpenEdition}

Journals

Édition électronique

URL : https://journals.openedition.org//ha/3190

DOI : 10.4000/lha.3190

ISSN : 1960-5994

Éditeur

Association Livraisons d'histoire de l'architecture - LHA

Référence électronique

Olivier Prisset, «Les grandes fortunes du cabinet Dauvergne (1850-1919), des conditions du

monopole aux limites d'un modèle économique », Livraisons de l'histoire de l'architecture [En ligne], 41 2021, mis en ligne le 15 juin 2021, consulté le 19 juin 2021. URL : http://journals.openedition.org//ha/ 3190 ; DOl : https://doi.org/10.4000//ha.3190

Ce document a été généré automatiquement le 19 juin 2021.

Tous droits réservés à l'Association LHA 


\section{Les grandes fortunes du cabinet Dauvergne (1850-1919), des conditions du monopole aux limites d'un modèle économique}

The great fortunes of cabinet Dauvergne (1850-1919), from monopoly conditions

to the limits of an economic model

Das Vermögen des Architekturbüros Dauvergne (1850-1919). Von den

Grundlagen eines Monopols zu den Grenzen einer Geschäftsmethode

\section{Olivier Prisset}

1 Parce qu'elle a été relativement discrète durant ses années d'activité, nous serions tentés d'écrire que la dynastie formée par l'architecte départemental de l'Indre Alfred Dauvergne (1824-1885) et ses deux fils Henry (1848-1917), qui prit sa suite, et Louis (1852-1937) qui posséda son propre cabinet parisien, est une famille oubliée de l'historiographie. Il est vrai qu'un cabinet, même prolifique, installé à Châteauroux au début du Second Empire était peu susceptible de prendre une place prépondérante dans les débats professionnels du temps. L'histoire de l'architecture pouvait à bon droit méconnaître leur œuvre, il était néanmoins impossible qu'elle les ignore tout à fait.

Dans l'Indre, département de deux cent quarante-trois communes, les Dauvergne ont disposé d'un terrain d'activité riche d'opportunités, En soixante-huit années de pratique, ils intervinrent près de deux mille fois à travers des chantiers d'ampleurs variées, allant de la construction de dizaines d'écoles communales jusqu'à la conception d'une usine de plus de huit hectares ${ }^{1}$. Confronté à pareil ouvrage, l'image communément admise de l'architecte départemental, homme de l'art isolé ${ }^{2}$ en terre inculte, faisant ses tournées à dos de mule sur des routes impraticables, est à interroger. Il est impensable qu'un praticien, même épaulé par ses fils lorsqu'ils attinrent l'âge d'intégrer son cabinet, soit parvenu à conserver la commande publique, ait pu s'attirer les faveurs d'un diocèse et conquérir la clientèle des plus grands 
propriétaires locaux sans l'appui d'une agence dûment formée. Or, comme le remarquait l'architecte Jules de Mérindol en 1864, "l'organisation d'une agence en province est une chose très difficile parce que l'on rencontre peu d'agents » ${ }^{3}$.

3 Ainsi, la réussite du cabinet Dauvergne offre-t-elle un bon moyen de documenter les stratégies adoptées par des architectes de la seconde moitié du XIX ${ }^{e}$ siècle. Les sources, même lacunaires ${ }^{4}$, susceptibles de faire percevoir le fonctionnement de leur agence sont d'autant plus précieuses que nous manquons de points de comparaisons avant l'instauration de la III ${ }^{e}$ République ${ }^{5}$.

\section{Le lieu de l'action}

4 Lorsqu'Alfred Dauvergne s'établit à Châteauroux vers 1849, quelque mois avant d'être nommé architecte départemental, il choisit d'installer son cabinet au sein de son habitation située au 5 rue des Pavillons, artère résidentielle plébiscitée par les notables de la Cité, dans un hôtel particulier que l'oncle de sa femme, le banquier Georges Bourdesol, avait fait construire quelques années auparavant (Ill.1). Ce dernier, qui possédait plusieurs terrains à travers la ville et avait participé à différentes opérations de lotissement ${ }^{6}$, venait de faire faillite. Dans cet hôtel, l'un des plus monumentaux de la ville, outre le couple Dauvergne et ses deux premiers fils, vivaient la mère de l'architecte, déjà veuve, sa sœur Louise Dauvergne, sa belle-mère MarieLouise Déribéré, ainsi que deux domestiques'. La distribution de l'hôtel de 1850 ne nous est pas parvenue avec exactitude, mais les archives montrent que le pavillon situé à gauche de la maison disposait d'une porte secondaire offrant un accès direct au cabinet de l'architecte.

\section{1 : G. G., façade principale de I'hôtel Bourdesol, Châteauroux}

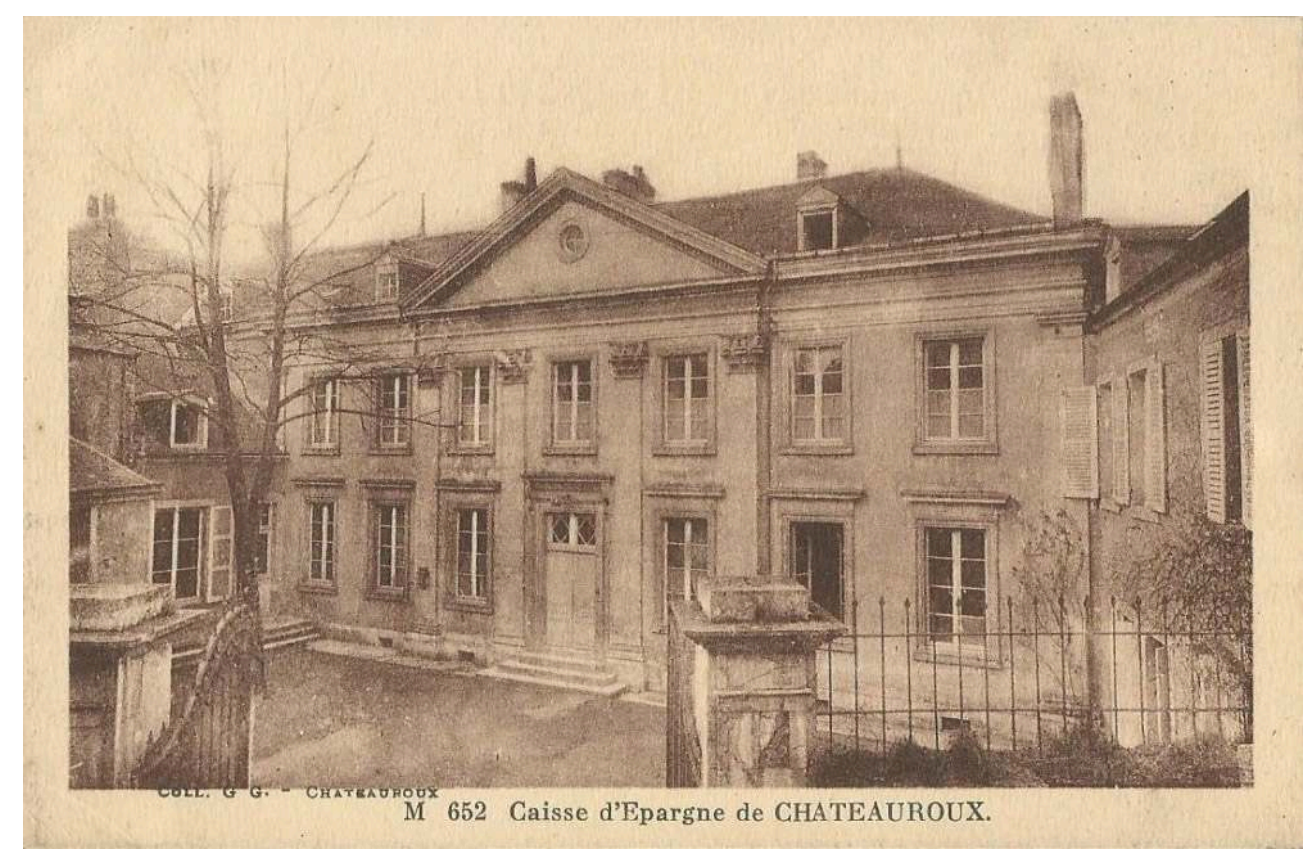

C.p.a., ca. 1920, collection particulière.

D. R. 
Cette présence de l'activité professionnelle dans le cadre familial pourrait surprendre dans le cas d'architectes officiant pour les pouvoirs publics. Toutefois, l'exercice à domicile parait avoir été la règle majoritaire jusqu'au milieu du XX siècle. Comme de nombreux autres "pères triomphants [et créateurs du XIX siècle qui ont] transformé leur maisonnée en atelier et leurs épouses et filles ou sœurs en secrétaires ${ }^{8}$, c'est en chef de famille qu'Alfred Dauvergne se lança à la conquête de l'Indre. Durant cette période de conquête, les différents membres de sa famille se placèrent au service de la vie de l'agence. Son épouse, notamment, parait avoir joué un rôle très actif dans l'entretien des «relations publiques» du cabinet, pour son mari puis pour son fils. En l'absence d'archives familiales, il est cependant difficile de déterminer le rôle exact joué dans la vie du cabinet par Léontine Dauvergne mais celui-ci est néanmoins indéniable dans la mesure où sa personne est régulièrement évoquée dans la correspondance liée aux travaux. À travers cette dernière, on s'aperçoit que Léontine Dauvergne entretenait les bons rapports de la famille notamment avec les clients récurrents de l'agence et leurs épouses. Toutefois, le fonctionnement de l'agence à ses débuts, si tant est que le terme d'agence ait pu alors être utilisé, n'a laissé aucune trace dans les archives car l'architecte décida rapidement de déménager.

Le 21 avril 1854', Alfred Dauvergne faisait l'acquisition de trois parcelles contiguës au parc de la Préfecture, situées sur le bord sud du plateau occupé par la ville historique. Il y érigea une maison dans laquelle il put emménager deux ans plus tard (Ill.2). L'architecte départemental s'était construit une maison bourgeoise adornée de motifs néo-renaissance. Son concurrent local le plus proche, l'architecte municipal Camille Létang (1851-1902), avait préféré lotir une parcelle, située rue de la République, à proximité des principaux pôles de la vie civique castelroussine, avec un immeuble de rapports pourvu de deux entrées (Ill. 3). Ainsi, dans ces deux cas, l'emplacement et les façades du cabinet reflétaient les catégories sociales de leurs clientèles respectives. Dauvergne cultivait l'image d'un confort discret dans les venelles du quartier de la Préfecture. Létang installait son domicile et son agence au cœur d'un quartier populaire qu'il allait contribuer à développer. 
III. 2 : façade principale de la maison Dauvergne

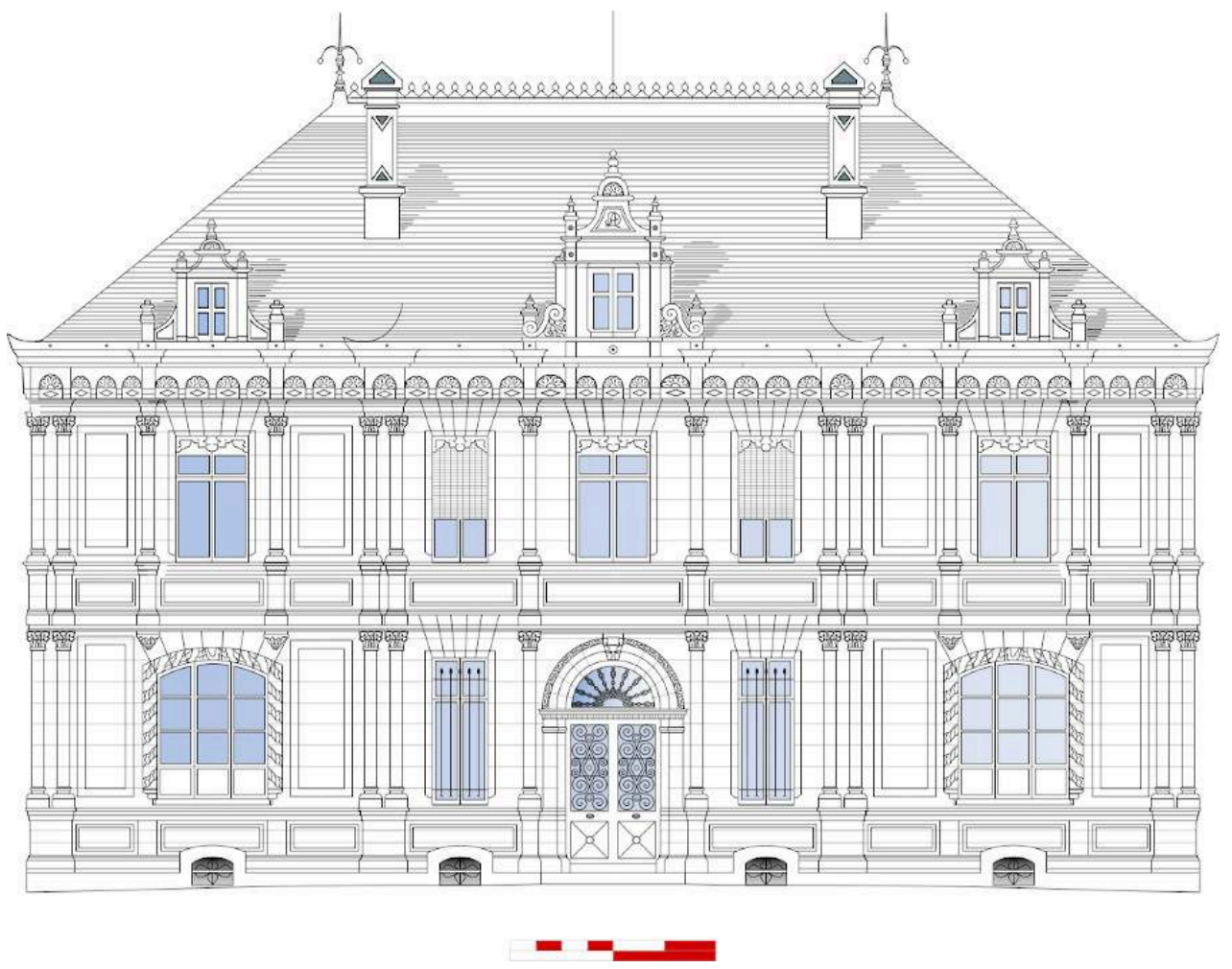

D’après une gravure publiée dans Compte rendu de la Société du Berry à Paris, août 1857, pl. 14.

Cl. Olivier Prisset

\section{3 : vue de l'immeuble occupé par le cabinet Létang à Châteauroux}

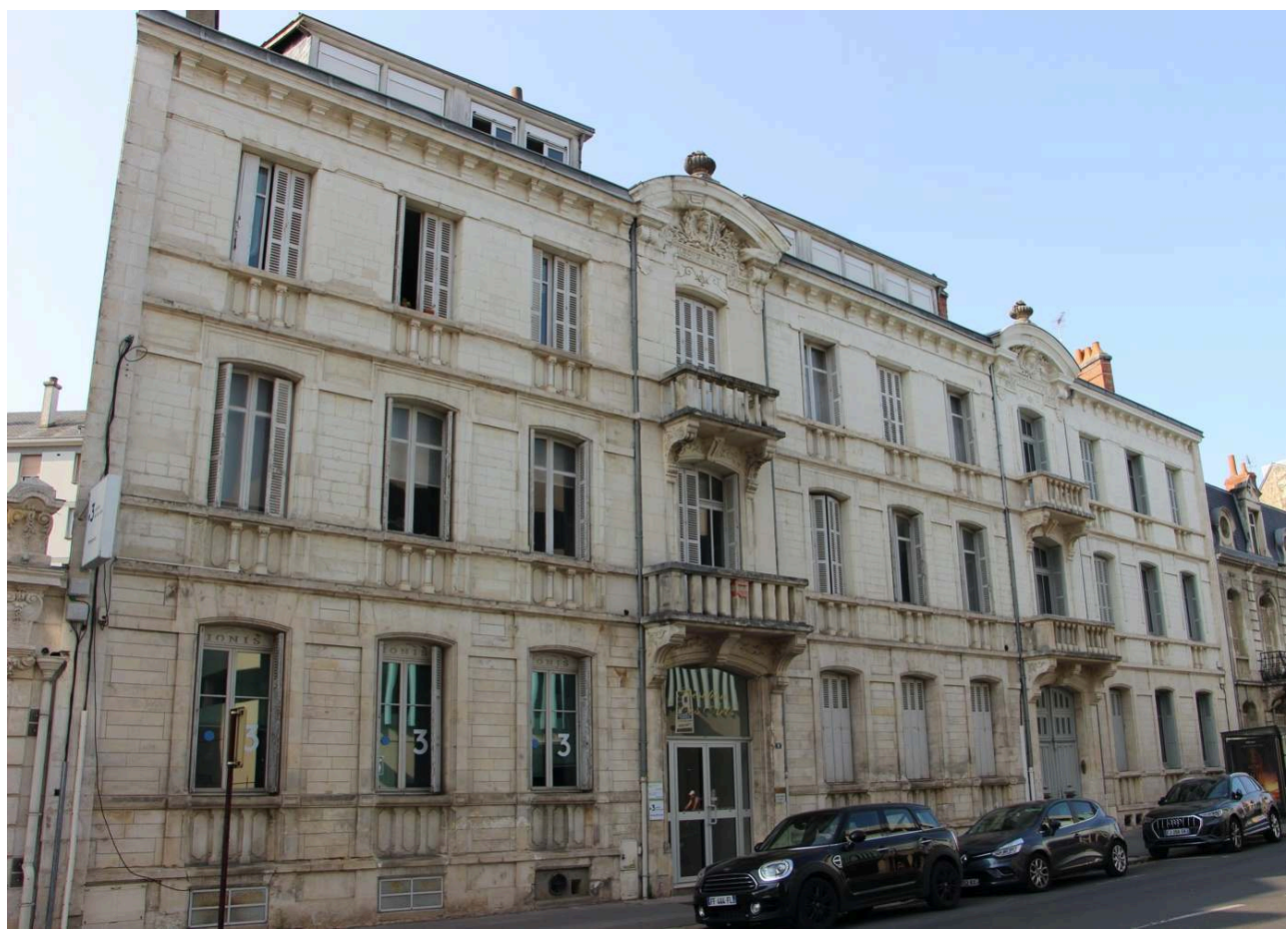

Durant le dernier quart du XIXe siècle, août 2020.

Cl. Olivier Prisset 
7 La maison Dauvergne se composait de trois niveaux. L'étage carré comprenait cinq chambres mais c'est au rez-de-chaussée, à côté des pièces de réception, qu'avaient été installés les bureaux de l'architecte, situés à droite de l'entrée principale. Le bureau des employés, donnait sur la cour d'entrée tandis que le cabinet ${ }^{10}$ de l'architecte avait vue sur le jardin ainsi que sur le quartier des Marins situé en contrebas. Le nombre de ses employés ne nous est pas connu mais l'inventaire ${ }^{11}$ établi dans le cadre de sa succession laisse imaginer le cadre de leur action. Passé le vestibule de la maison, un couloir donnait accès au bureau dévolu à l'agence, occupé par le métreur-vérificateur ainsi qu'un ou deux dessinateurs. Celui-ci comportait deux tables sur tréteaux entourées de six chaises et dix-huit planches à dessin y était entreposées ; un calorifère avait été logé dans la cheminée. En cela, les employés du cabinet Dauvergne semblent avoir été mieux traités que leurs confrères parisiens ${ }^{12}$. Aucune bibliothèque n'est mentionnée mais des placards furent aménagés lors de l'agrandissement du bureau par Henry Dauvergne durant le dernier quart $\mathrm{du}$ siècle. Comparé aux plans d'autres agences contemporaines $^{13}$, celle des Dauvergne était de taille réduite. Il est vrai que les très rares cabinets d'architectes provinciaux qui nous sont connus pour cette période étaient installés dans des villes plus développées que Châteauroux, où la commande était plus soutenue que dans ce territoire rural.

Cette sphère dédiée au personnel, à l'instar d'un espace ancillaire, disposait d'un accès dédié, reporté sur la façade latérale, côté ouest, $\mathrm{Au}$ début du $\mathrm{XX}^{\mathrm{e}}$ siècle, suivant une tendance générale ${ }^{14}$, Henry Dauvergne choisit de séparer les espaces domestiques de l'activité de l'agence. Il accentua l'indépendance de cette dernière vis-à-vis du reste de la maison, avec la création d'un vestibule au-devant de l'entrée des employés.

9 L'inventaire du cabinet d'Alfred Dauvergne correspond à l'imaginaire attaché aux bureaux d'architectes du XIX ${ }^{\mathrm{e}}$ siècle. Ce cabinet de travail était l'espace de réception des commanditaires, des entrepreneurs et des solliciteurs de toute nature. Il devait donc, et peut-être avant tout, être agencé pour recevoir la clientèle dans un environnement confortable, le salon pouvant même faire office d'antichambre. L'incontournable table sur tréteaux était installée au sud de la pièce. Elle voisinait avec la bibliothèque professionnelle $\mathrm{du}$ cabinet, une statuette en terre cuite et quinze plâtres, copies d'éléments d'architecture. Cette pièce composait un cadre flatteur qui vendait davantage l'idée de l'architecte au travail plutôt que celle d'un espace de création effectif. Par ailleurs, le grand nombre de sièges présents dans la pièce retient l'attention. On dénombre en effet six chaises, trois fauteuils, une chauffeuse, une fumeuse et, dans une alcôve installée à l'opposé de la table, un divan avec cinq coussins; soit à peine moins que dans le salon familial contigu ${ }^{15}$.

Malgré ce potentiel de représentation, il semble que l'activité de l'agence et plus généralement celle des commis de l'architecte ${ }^{16}$, ait été le plus souvent cantonnée à un ouvrage discret quoique tout à fait crucial. Malgré son installation en façade de la maison, la pièce occupée par l'agence n'était pas sur le chemin que devaient emprunter les clients avant de pénétrer dans le bureau de l'architecte. Pourtant, l'activité de ses occupants était indispensable à des architectes aussi productifs que le furent les Dauvergne. 


\section{Le statut ambivalent de l'agence}

11 Une fois nommé, l'architecte départemental était seul à rendre des comptes à l'administration et il lui incombait, le cas échéant, de se doter d'une agence de collaborateurs qu'il rémunérerait sur ses honoraires ${ }^{17}$. En cela, le cabinet Dauvergne entretint moins la confusion des genres que certains confrères attachés au service d'autres préfectures ${ }^{18}$ puisqu'il n'usait pas d'un bureau au sein de la préfecture et préféra disposer de ses propres locaux installés au sein de la demeure familiale. Toutefois, sans livres de gestion conservés, ni d'archives comptables ${ }^{19}$, il nous a été impossible d'évaluer le nombre d'employés ou d'estimer le rythme de leur rotation.

Pour les architectes, l'enjeu était de parvenir à s'entourer de dessinateurs efficaces et productifs tout autant que d'hommes accoutumés aux travaux techniques inhérents à la maîtrise d'ouvrage, depuis la production de relevés architecturaux jusqu'à la tenue de carnets d'attachements, et aux contingences d'une surveillance de chantier. À Paris, Louis Dauvergne pouvait puiser sa main-d'œuvre auprès des élèves de l'École des beaux-arts ${ }^{20}$ qui décidaient de « faire la place » dans une agence ${ }^{21}$. Mais, durant toute la période d'activité du cabinet, les candidats répondant à toutes ces compétences ne furent pas nombreux en dehors des principaux centres urbains.

D'ailleurs, la carrière de commis pouvait manquer d'attrait. Régulièrement, les architectes, tout à leur quête d'une reconnaissance officielle et publique de leur profession, préféraient apparaître en personnages omnipotents. Dans leur quête d'un statut officiel et d'une reconnaissance professionnelle, ils furent enclins à minimiser la présence physique de leur agence. Les employés constituaient l'agence ; Alfred et Henry Dauvergne incarnaient le cabinet. Leur partenariat était donc le versant public d'une officine occupée par une population de collaborateurs au statut parfois précaire et cantonnés à une pratique anonyme.

14 L'employé le plus identifiable du cabinet fut également le plus fidèle collaborateur des Dauvergne. François-Élie Moreau (inc.), dont nous pouvons attester l'activité entre 1856 et 1895, a, semble-t-il, commencé à travailler pour Alfred Dauvergne durant les premiers temps de son installation à Châteauroux. En 1870, c'est comme premier commis qu'il signait un procès-verbal de réception au nom de l'architecte ${ }^{22}$. C'est encore lui qui en janvier 1869 servit de prête-nom à son employeur lorsque celui-ci, conseiller municipal de Tendu, accepta d'y construire une école. Ce dernier ne pouvait pas être maitre d'œuvre dans une commune dont il était édile mais rien n'interdisait à son employé d'endosser cette responsabilitée ${ }^{3}$. Après 1870 , le premier commis fut le seul employé du cabinet qui obtint d'être cité nommément dans les courriers de certains des commanditaires $\mathrm{du}$ cabinet $^{24}$. En plus d'une place prépondérante dans le fonctionnement de l'agence, ce collaborateur zélé et efficace parait avoir atteint un bon degré d'expertise. Et, en 1893, c'est en tant qu'architecte, et non plus comme simple commis de son ancien patron, qu'il se chargea de signer la déclaration de décès du fils d'Henry Dauvergne ${ }^{25}$.

15 L'ultime premier commis de l'agence laissa également une trace significative dans les archives Dauvergne. Henri Virault (inc.), mentionné à partir de 1889, survécut à Henry Dauvergne et assura l'intérim de l'agence aux côtés de Louis Dauvergne. Il caressa même l'idée de lui racheter le cabinet. Malheureusement, la nature exacte de son action est difficile à appréhender à partir des documents épars de cette période de transition. 
D'autres employés et apprentis demeurent anonymes lorsqu'ils sont évoqués dans les correspondances. Leur nombre et leurs âges ont pu évoluer en fonction des besoins du cabinet et des difficultés de recrutement. De même, leur passage au sein de l'agence pouvait ne durer que le temps de leur formation. L'apprentissage reproduisait les méthodes de l'école des Beaux-arts. L'agence était un lieu d'émulation où les jeunes arrivants se formaient par imitation. Les anciens concours scolaires d'Alfred Dauvergne (Ill.4) servaient de sujets d'étude pour les novices (Ill.5). Bien que nous manquions d'informations à leur égard, un de ces élèves passagers mérite néanmoins d'être évoqué : Emmanuel Lansyer (1835-1893), futur peintre paysagiste, se morfondait à la perspective de devenir notaire. En 1855, afin d'approcher la carrière artistique, il convainquit ses parents de l'envoyer faire ses classes chez son cousin Alfred Dauvergne. Il passa deux années à Châteauroux, y acquit les bases de la pratique avant de partir pour Paris où il intégra, non l'École des beaux-arts mais l'atelier d'Eugène Viollet-le$\operatorname{Duc}^{26}$.

\section{4 : Alfred Dauvergne, [Façade principale d'un temple et de son nymphée]}

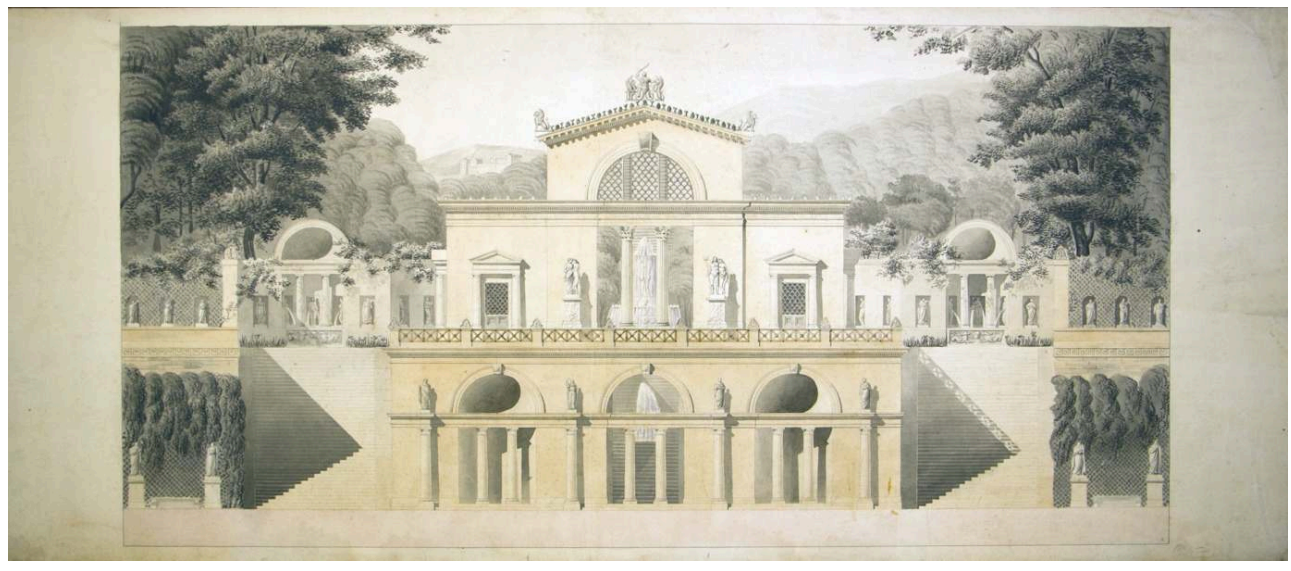

ca. 1847, 70,3 × 30,2 cm, encres sur papier vergé, AD36 n.c.

Cl. Olivier Prisset

III. 5 : anonyme, [Façade principale d'un temple et de son nymphée]

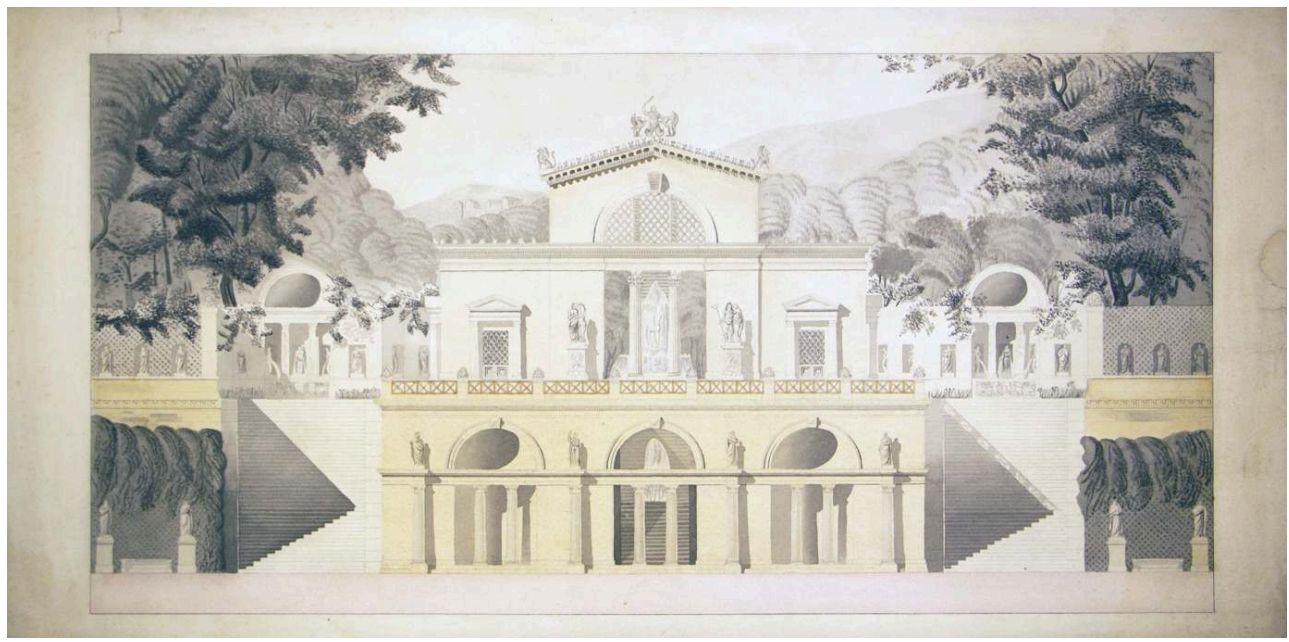

N. d., 70,3 × 30,4 cm, encres sur papier vergé, AD36 n.c.

Cl. Olivier Prisset. 


\section{La ligne de l'agence, de l'efficacité au confort}

La bonne volonté et d'éventuelles prédispositions pour le dessin ne pouvaient compenser les lacunes d'une formation parcellaire. Plusieurs années s'écoulaient avant que ces commis formés au gré des chantiers acquièrent suffisamment d'autonomie pour pouvoir prétendre suppléer une absence de l'architecte. Cela sans parler de dresser des projets par eux-mêmes ${ }^{27}$. Ce manque de flexibilité était compensé par la pratique d'une architecture sérielle que la commande publique appelait de ses vœux ${ }^{28}$ et que les Dauvergne adoptèrent pleinement. Ils l'appliquèrent même précocement à leur clientèle privée ainsi qu'en attestent les châteaux de Laborde (Val-Fouzon) (Ill. 6) et de Rezay (Mâron) (Ill. 7) tous deux construits autour de 1860.

\section{6 : vue de la façade sud-est du château de Laborde}

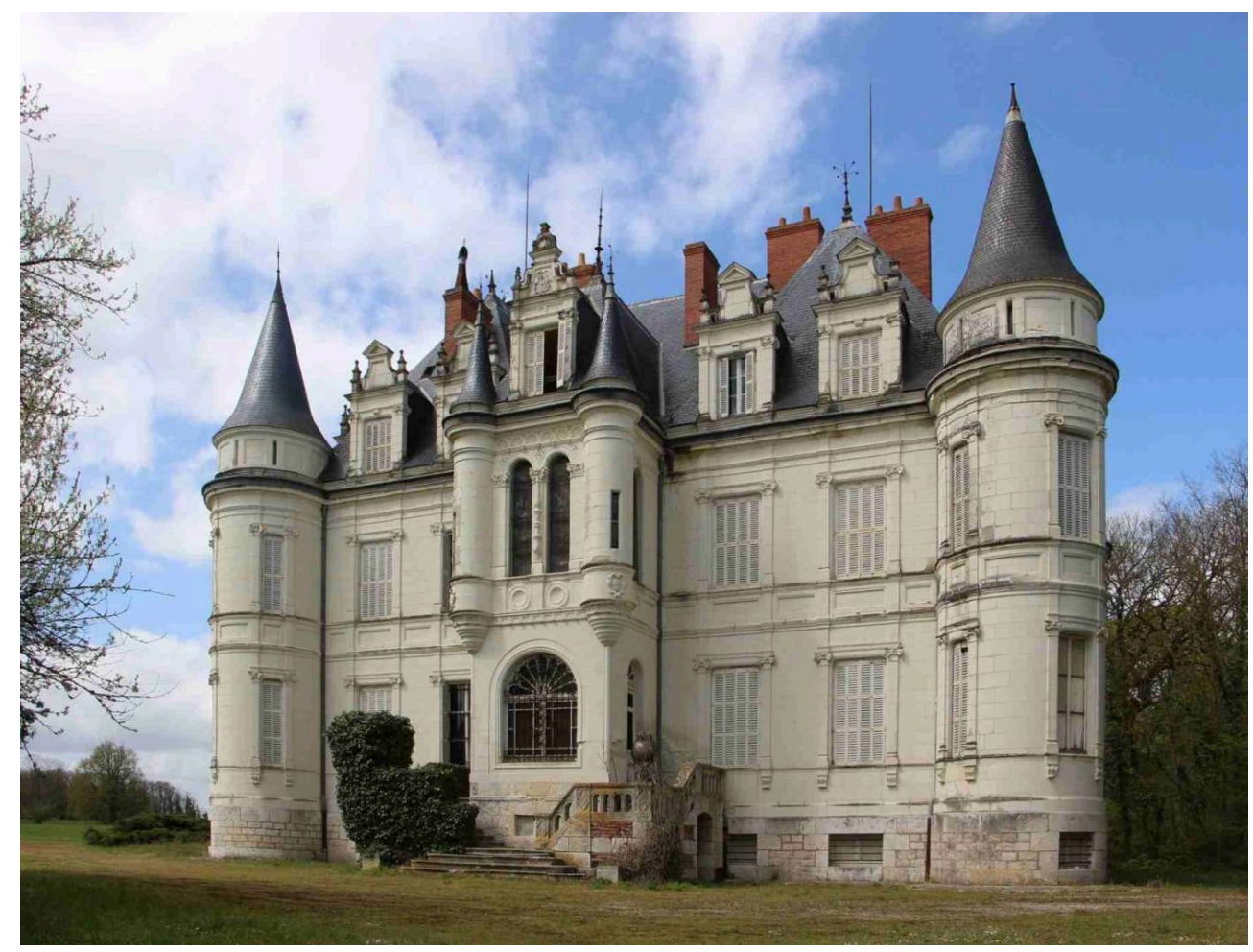

Val-Fouzon, construit par Alfred Dauvergne entre 1862 et 1865, avril 2018.

Cl. Olivier Prisset 


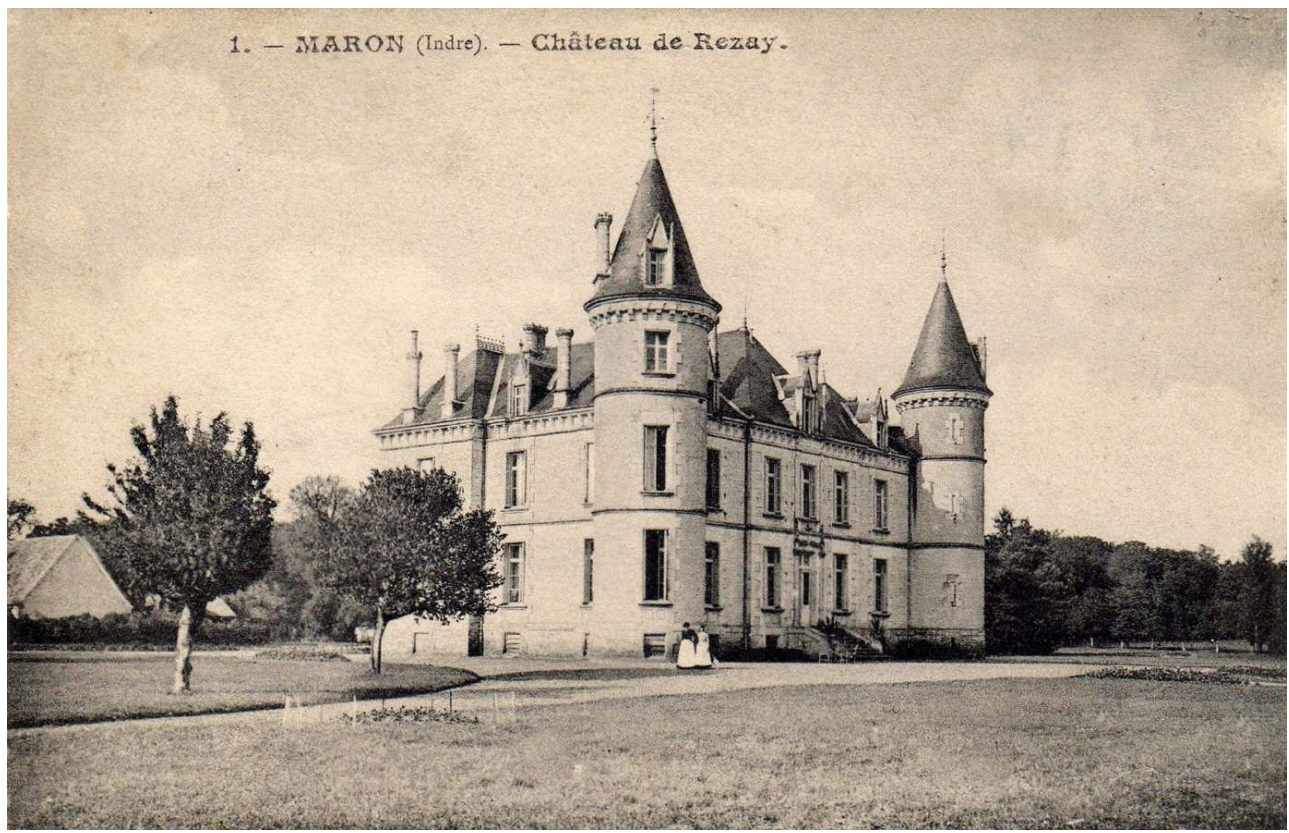

Depuis le sud (Mâron), ca. 1910, c.p.a, 9 × 15 cm, collection particulière.

D. R.

En procédant ainsi, le cabinet se dotait d'une réactivité unique dans ce département et occupa une position prépondérante qui lui resta indisputée pendant une trentaine d'années. Entre 1876, date à laquelle Henry Dauvergne obtint son diplôme et 1884, année du départ en retraite de son père, le cabinet comptabilisa une moyenne annuelle de trente commandes d'importance, que l'itération de ses schémas lui permit d'honorer à la grande satisfaction des aménageurs locaux et des propriétaires qui purent ainsi se doter d'ensembles homogènes de bâtiments d'usage et de villégiature. La multiplication de ces interventions à l'échelle de l'Indre ${ }^{29}$ imposa une tendance que les entrepreneurs locaux tentèrent de reproduire ${ }^{30}$. Cependant, avec le succès vint la lassitude. L'unicité des solutions offertes par l'agence castelroussine, ainsi qu'une plus forte concurrence locale, détournèrent insensiblement sa clientèle particulière vers la demi-douzaine d'architectes qui s'installèrent en Berry entre 1876 et 1914.

Henry Dauvergne, en dédaignant de faire évoluer la ligne de son cabinet, se confina dans une perpétuation stylistique contre-productive et manqua le tournant architectural de la fin du siècle. Il conserva une activité rémunératrice mais les dernières décennies de sa carrière lui apportèrent moins de commandes prestigieuses. En 1898, l'effondrement de la flèche de l'église Saint-Germain de La Châtre, qu'il avait achevée huit jours auparavant, à quoi s'ajouta la banqueroute de son frère en juin 1902, actèrent le déclin des activités la dynastie. Afin de solder les dégâts dont il était responsable, l'architecte départemental continua d'œuvrer et maintint l'activité de son agence jusqu'à sa mort en 1917. Son frère endossa l'intérim jusqu'à la fin du conflit puis vendit l'agence à un ancien concurrent.

Les nombreux bâtiments qu'ils essaimèrent durant soixante-dix années témoignent, aujourd'hui encore, de leurs qualités de bâtisseur et attestent de leur habileté à répondre aux attentes et aux besoins de leurs contemporains. Cette œuvre collective et méthodique, véritable monument de patience, illustre de manière exemplaire les 
enjeux auxquels furent confrontés nombre d'architectes installés dans les départements français du second XIX ${ }^{\mathrm{e}}$ siècle. La chance des Dauvergne fut que leurs archives parviennent à être conservées en un ensemble cohérent et exhaustif.

\section{NOTES}

1. L'usine de draps que l'industriel Pierre Balsan (1807-1869) fit construire à Châteauroux entre 1859 et 1864 .

2. Nolwenn Rannou, Joseph Bigot (1807-1894), architecte et restaurateur, Rennes, Presses universitaires de Rennes, 2006, p. 79.

3. Jean-Michel Leniaud, Les cathédrales au XIXe siècle, Paris, Economica, 1993, p. 689.

4. Cet article, tiré sur une thèse de doctorat en cours d'achèvement, s'appuie sur le dépouillement du fonds d'archives du cabinet Dauvergne conservé aux Archives départementales de l'Indre (AD36) sous la cote $24 \mathrm{~J}$, fonds Dauvergne/Bouguin.

5. Dans les revues professionnelles qu'il a dépouillées, Maxime Decommer constate la rareté des recensions et l'absence de plans concernant des cabinet d'architectes avant $1879 c f$. Maxime Decommer, Les architectes au travail, Rennes, Presses universitaires de Rennes, 2017, p. 225-234.

6. Annabelle Paillisson, Création et urbanisation de la rue de la Gare de Châteauroux, mémoire de maîtrise : histoire de l'art, Tours, université François-Rabelais, 2001, vol. 1, p. 29-31.

7. Minutes du Recensement de Châteauroux, 1851, p. 153-154 [AD36, M 4677].

8. Michelle Perrot, La vie de famille au XIX ${ }^{e}$ siècle, Paris, Éditions du Seuil, 2015, p. 49.

9. Étude de $\mathrm{M}^{\mathrm{e}}$ Villepelet, Donation-partage par M. et Mme Dauvergne à leurs deux fils, 21 juillet 1884, fol. 4 [AD36 2E 10737].

10. Au XIX ${ }^{\mathrm{e}}$ siècle, le terme de cabinet d'architecte désigne le bureau, lieu de travail personnel de l'architecte, plus que la totalité de son entreprise. Lorsque l'architecte n'était pas seul à la tête de l'agence, ainsi que ce fut le cas avec le partenariat d'Alfred et d'Henry Dauvergne, ce mot sert également à distinguer les architectes de leurs employés, renvoyés à l'anonymat de l'agence.

11. Étude de $\mathrm{M}^{\mathrm{e}}$ Villepelet, Donation-partage par M. et Mme Dauvergne à leurs deux fils, 21 juillet 1884, fol. 4 [AD36 2E 10737].

12. Maxime Decommer, op. cit., p. 235-250.

13. Archives d'architecture de la Loire, Les Lamaizière, architectes à Saint-Étienne 1880-1925, SaintÉtienne, Publications de l'université de Saint-Étienne, 1995, p. 13.

14. Dominique Eleb et Anne Debarre, L'invention de l'habitation moderne, Paris 1880-1914, Paris, Hazan/A.A.M., 1995, p. 24.

15. En comparaison, le salon contenait six chaises volantes, deux poufs, un canapé et ses six fauteuils, une chaise longue et, au surplus, un tabouret pour le piano Érard.

16. Maxime Decommer, op. cit., p. 250-254.

17. Chantal Callais, À corps perdu, Pierre-Théophile Segretain architecte (1798-1864), les architectes et la fonction publique d'État au XIXe siècle, La Crèche, Geste éditions, 2010, p. 225.

18. Sophie Nasi, Louis Sauvageot (1842-1908) architecte et restaurateur à Rouen, Rennes, Presses universitaires de Rennes, 2010, p. 73-74.

19. Nous fondons notre analyse sur une vingtaine de pièces de correspondance conservées sous différentes cotes du fonds $24 \mathrm{~J}$ ainsi que sur quelques documents paraphés par leurs commis. 
20. Le dossier d'élève de Charles Cravio conserve un certificat de travail délivré le 17 décembre 1892 par Louis Dauvergne. L'élève architecte avait travaillé au sein de son agence entre 1890 et 1892 cf. Marie-Laure Crosnier Leconte, Dossier d'élève à l'École des Beaux-arts de Paris et biographie de Charles Cravio, https://agorha.inha.fr/inhaprod/ark:/54721/00279913, [dernière consultation le 13 janvier 2020].

21. Jean-Pierre Martinon, Traces d'architectes, éducation et carrières d'architectes Grand-Prix de Rome aux XIX ${ }^{e}$ et XX $X^{e}$ siècles en France, Paris, Anthropos, 2001, p. 10-12.

22. Mémoire des travaux exécutés chez M. Nuret, libraire, par Marteau maître maçon pour la construction d'un bâtiment en 1869, 4 juillet 1870 [AD36 $24 \mathrm{~J} 381$ ].

23. Élie Moreau, Commune de Tendu, projet de construction d'une école et mairie, 20 janvier 1869, crayon, encre et lavis sur papier calque, 50 x $72 \mathrm{~cm}$ [AD36 24 J 191].

24. Voir, par exemple, la Lettre de Jules Frichon de Vorys adressée à Alfred Dauvergne le 12 octobre 1873 [AD36 24 J 583].

25. Registre d'état civil de Châteauroux, décès de l'année 1893, n 502, 13 décembre 1893 [AmC 1E 223].

26. Emmanuel Lansyer, Autobiographie, n. d., p. 5 [Ville de Loches-Collection Lansyer].

27. Au regard des archives conservées, seul le premier commis bénéficiait de quelque autonomie et était autorisé à établir des avant-projets à la demande de son patron.

28. Jean-Michel Leniaud, 1993, op. cit., p. 484-486.

29. Seules vingt-cinq communes échappèrent au cabinet Dauvergne soit $10 \%$ du territoire de l'Indre.

30. Hélène Guéné et François Loyer, L'Église, l'État et les architectes, Paris, Éditions Norma, 1995, p. 16.

\section{RÉSUMÉS}

Comment un architecte, ancien élève de l'École des Beaux-arts de Paris, faisait-il carrière dans la France de 1850 lorsque sa pratique était implantée en province? De quelle manière, trente ans plus tard, une fois fortune faite, parvenait-il à transmettre sa clientèle à ses fils afin qu'ils puissent assurer sa succession? Comment évoquer le cadre de leur activité, le profil de leurs employés et les stratégies professionnelles qu'ils parvinrent à développer quand les comparaisons sont rares et demeurent globalement méconnus des chercheurs ? Voici, résumés en quelques lignes, certains des enjeux offerts par l'étude d'une dynastie telle que celle que formèrent Alfred (1824-1885), Henry (1848-1917) et Louis Dauvergne (1852-1937). En plus de constituer une œuvre symptomatique et pléthorique, le cas de cette famille permet de mieux comprendre le fonctionnement des agences d'architectes implantés dans les départements français durant cette période.

How did an architect, a graduate of the École des Beaux-arts, make a career in in the French provinces in the middle of the 19th century? How, thirty years later, once his fortune had been made, did he pass on his clientele to his sons? With few existing studies to draw on, what tools are available to the historian seeking to understand the conditions and context of the work of these architects, the background of their employees and the professional strategies they developed? These are some of the questions raised by the study of the "dynasty" of architects formed by Alfred (1824-1885), Henry (1848-1917) and Louis Dauvergne (1852-1937). The example 
of this practice that produced an abundant body of work characteristic of the period helps us to better understand the ways that architectural firms in the French provinces functioned at that time.

Auf welche Weise ist es einem an der École des Beaux-Arts in Paris ausgebildeten Architekten gelungen, im Frankreich der zweiten Hälfte des 19. Jahrhunderts eine Karriere aufzubauen, wenngleich sich der Schwerpunkt seiner Geschäftstätigkeit in der entlegeneren Provinz befand? Und auf welchem Wege erreichte er es dreißig Jahre später als wohlhabender Geschäftsmann, seine Kundschaft an seine Söhne zu übergeben und seine Nachfolge zu sichern? Mit welchen Mitteln kann schließlich eine Recherche zur Tätigkeit dieser Akteure durchgeführt werden, zum Profil ihrer Mitarbeiter und $\mathrm{zu}$ ihren beruflichen Strategien, wenn die Forschung kaum Vergleichsbeispiele kennt? Dies sind einige der Leitfragen, denen in diesem Beitrag am Beispiel der Architektendynastie der Familie Dauvergne nachgegangen werden soll: Alfred (1824-1885), Henry (1848-1917) und Louis (1852-1937). Über ihr beispielhaftes und reichhaltiges Werk hinaus erlaubt es die Studie der Familie Dauvergne, Aussagen zum Betrieb von Architekturbüros dieser Zeit in den französischen Departements zu treffen.

\section{INDEX}

Mots-clés : agence d'architecture, dynastie, XIXe siècle, architecture publique, architecture privée, architectes départementaux, histoire économique, histoire sociale

\section{AUTEUR}

\section{OLIVIER PRISSET}

Olivier Prisset a fait ses études en histoire de l'art et histoire de l'architecture contemporaine à l'université de Tours où il a également été chargé de cours. Après avoir étudié, durant son Master, les restaurations pratiquées au château d'Azay-le-Rideau (Indre-et-Loire) durant le $\mathrm{XIX}^{\mathrm{e}}$ siècle, il mène actuellement une thèse de doctorat sur la dynastie des architectes Dauvergne (1824-1937), sous la direction de Jean-Baptiste Minnaert. Adresse électronique :

olivier.prisset@outlook.com 